\title{
Endovascular therapy after stroke in a patient treated with dabigatran
}

\section{Andrew W Moey \\ MB BS \\ Stroke Fellow \\ Simon A Koblar \\ MB BS, FRACP, PhD, \\ Neurologist and \\ Associate Professor \\ Steve Chryssidis \\ MB BS, RANZCR \\ Radiologist $^{1}$ \\ Martin Robinson MB BS, FRACP, \\ Neurologist and \\ Head of Neurology Unit \\ Jim Jannes \\ MB BS, FRACP, PhD \\ Neurologist and \\ Head of Stroke Unit}

1 Queen Elizabeth Hospital Adelaide, SA.

2 Stroke Research Programme,

University of Adelaide Adelaide, SA.

awomoey@gmail.com

MJA 2012; 196: 469-471 doi: 10.5694/mjal1.11617

Editorials p 431, p433

\begin{abstract}
This is the first report of endovascular therapy for acute ischaemic stroke in a patient with atrial fibrillation who was taking the direct thrombin inhibitor dabigatran for stroke prevention. As more of these agents will become available soon, endovascular therapy is likely to be used more frequently for acute stroke.
\end{abstract}

\section{Clinical record}

We report the case of a 76-year-old right-handed independent woman with known atrial fibrillation managed with dabigatran prophylaxis (110 mg twice daily) for several months, who had taken her last dose of dabigatran 30 minutes before the onset of stroke symptoms. She presented to the emergency department 1.5 hours after symptom onset with an acute left hemiplegia, left hemianaesthesia and left homonymous hemianopia corresponding to a National Institutes of Health Stroke Scale (NIHSS) score of 18.

The local hospital acute stroke treatment protocol was followed, commencing with attendance by a code stroke team. Conventional non-contrast computed tomography (CT) of the brain performed 2 hours after symptom onset showed subtle and early obscuration of the right posterior insular cortex (Box 1). Findings on perfusion CT (Aquilion One, Toshiba Medical Systems, Otawara, Japan) were consistent with a large area of ischaemic penumbra in a

1 Initial non-contrast computed tomography scan performed 2 hours after symptom onset

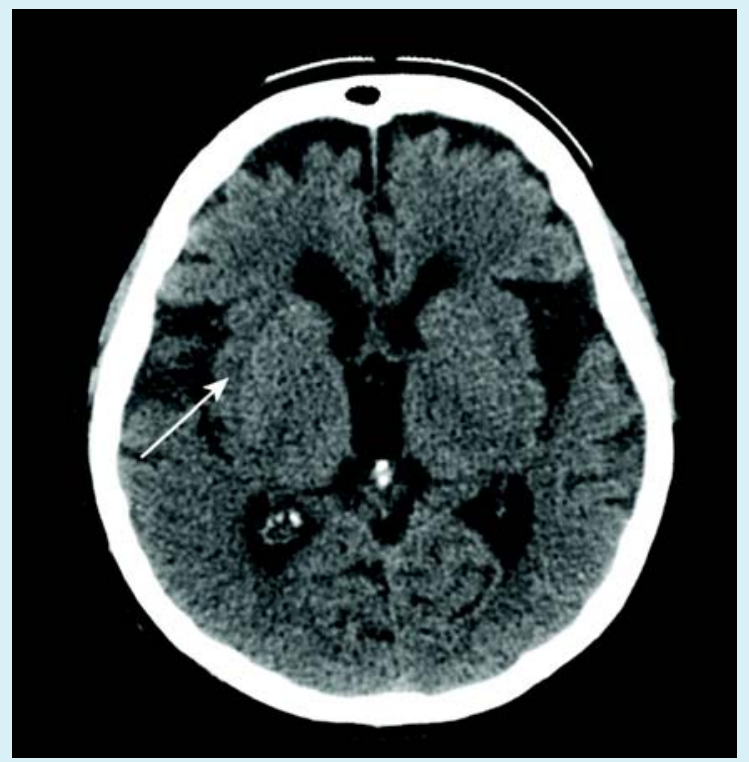

Image shows subtle and early obscuration of the right posterior insular cortex with preserved basal ganglia detail. right posterior middle cerebral artery (MCA) distribution (Box 2), corresponding to a right MCA (M2 segment) thrombotic occlusion on CT angiography.

The platelet count was $206 \times 10^{9} / \mathrm{L}$ (reference interval [RI], $\left.150-400 \times 10^{9} / \mathrm{L}\right)$, activated partial thromboplastin time (aPTT) was $33 \mathrm{~s}$ (RI, 24-38 s) and international normalised ratio (INR) was 1.3 (RI, 0.9-1.2). The blood glucose level was $6.8 \mathrm{mmol} / \mathrm{L}$ (RI, 3.2-5.5 mmol/L). On the basis of the exclusion criterion of current oral anticoagulant therapy, intravenous recombinant tissue plasminogen activator (rtPA) was contraindicated. Therefore, as the patient met the local hospital inclusion criteria for endovascular therapy (ischaemic stroke with contraindication to intravenous tissue plasminogen activator within 3-6 hours, NIHSS score $>4$, less than a third MCA territory core infarction apparent on a CT scan of the brain and CT perfusion, and able to provide informed consent) this procedure was commenced at 4 hours and 15 minutes after symptom onset. The patient's blood pressure before the procedure was $178 / 95 \mathrm{mmHg}$, and thus was lowered with propofol to $140 / 65 \mathrm{mmHg}$.

A clot retrieval device (Solitaire AB Neurovascular Remodeling Device, $4 \times 15 \mathrm{~mm}$; ev3 Inc, Irvine, Calif, USA) was used to achieve reperfusion to the right MCA beyond the M2 segment (Box 3). The procedure was performed under general anaesthesia. Initial diagnostic imaging was performed using a 5-French diagnostic catheter and heparin (3000 IU) was delivered intravenously. A 6-French shuttle sheath (Cook Medical, Brisbane, Qld) was positioned within the common carotid artery, and then a 6-French neuron guide catheter was positioned within the inferior right internal carotid artery. A Transcend floppy guidewire (0.014"; Boston Scientific, Natick, Mass, USA) and Rebar 18 microcatheter (ev3 Inc, Irvine, Calif, USA) were advanced into the right MCA and two occluded branches selected. The microguidewire was passed through the thrombus and the microcatheter was advanced over the wire about $5 \mathrm{~mm}$ beyond the thrombus. The position of the microcatheter was confirmed by contrast injection. The stent was then advanced to the distal tip of the microcatheter and then unsheathed, both within and just beyond the thrombus. The distal microcatheter marker was positioned proximal to the uncovered stent. The unsheathed Solitaire $\mathrm{AB}$ stent and microcatheter were removed as a unit while suction was applied to the guiding catheter. This procedure was repeated with each pass. Angiographic assessment was performed 


\section{Initial perfusion computed tomography scan}

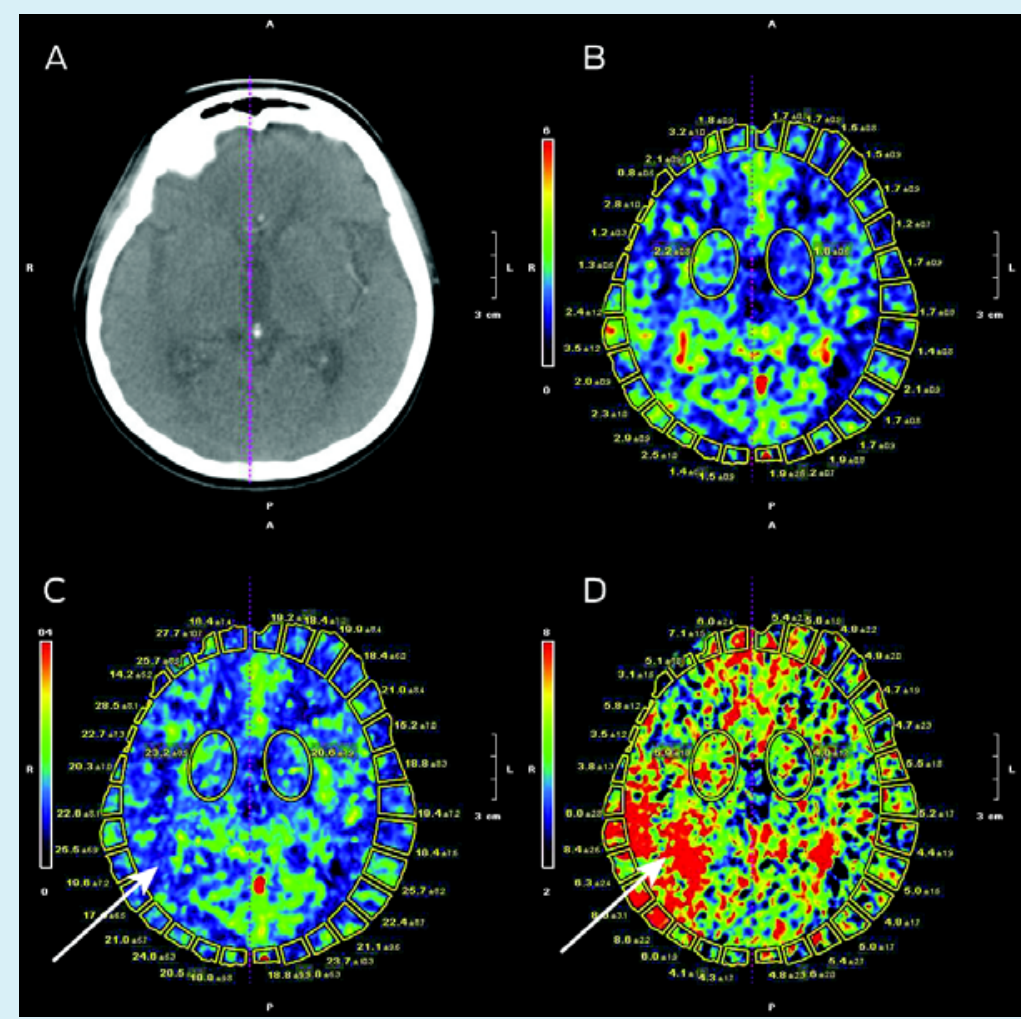

The scans show: $(A)$ a plain CT scan; (B) a symmetrical cerebral blood volume map; (C) slight flow reduction in the right temporoparietal junction on the cerebral blood flow map; and $(D)$ a corresponding area of abnormality on the mean transit time map.

\section{Angiographic imaging before and after the endovascular procedure}
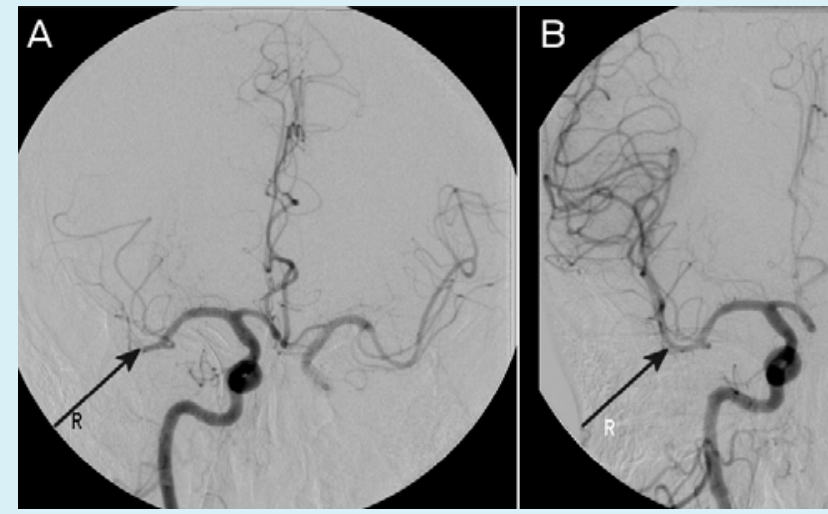

(A) Right M2 segment middle cerebral artery occlusion (arrow)

(B) Reperfusion achieved after mechanical clot

after each pass with the Solitaire $\mathrm{AB}$ stent to assess for revascularisation status, vessel trauma or distal emboli. Two passes as described using the Solitaire $A B$ stent were performed within each of the two affected vessels achieving TIMI (Thrombolysis In Myocardial Infarction) grade 2 flow or better.

Contrast extravasation was seen arising from the posterior branch of the right MCA on a subsequent contrast digital subtraction angiogram (DSA). However, this was not seen on the final contrast cerebral DSA. Vessel morphology remained normal on final imaging. Despite the extravasation seen, no clinical deterioration was observed. Right groin haemostasis was achieved using a 6-French Angio-Seal artery closure device. The duration of the procedure was 2 hours, and recanalisation was achieved at 5.5 hours after symptom onset. Conservative management was chosen after consultation with the neurosurgical team, and the patient was monitored in the intensive care unit after the procedure.

The NIHSS scores immediately after the procedure and at 24 hours were 17 and 8, respectively. A routine CT scan of the brain at 24 hours showed hyperdensity in the subarachnoid space (which had largely resolved on further CT imaging by Day 11) as well as infarction, predominantly within the posterior insular cortex (Box 4).

Over the following week, the patient showed clinical improvement with resolution of hemiparesis and only residual left hemisensory and visual inattention at the time of discharge to rehabilitation.

Owing to the clinical improvement and the clearing of the CT hyperdensity, it was felt that repeat angiography of the cerebral vessels would be unjustified. After the Day 11 CT scan, secondary stroke prevention therapy with warfarin (which replaced dabigatran) was started with the patient's consent. At 3-month follow-up, the patient's modified Rankin Scale score was 1, and she had returned to independent living at home.

\section{Discussion}

Intravenous administration of rtPA is now considered standard therapy in acute stroke, and is being widely used around the world and in Australia. ${ }^{1}$ However, the concomitant use of anticoagulants, including dabigatran, contraindicates use of rtPA because of a theoretical risk of intracerebral haemorrhage. Despite this, two recent case reports have shown benefit of using rtPA in patients taking dabigatran who have had a stroke, although its safety requires confirmation in a larger cohort. ${ }^{2,3}$ Endovascular approaches using mechanical clot extraction devices do not expose patients to thrombolytic agents, and thus their use may have fewer haemorrhagic sequelae in stroke patients taking dabigatran. ${ }^{4}$

To the best of our knowledge, this is the first case report of endovascular therapy for acute ischaemic stroke in a patient taking dabigatran. This situation is likely to occur more frequently in the near future as direct thrombin inhibitors including dabigatran become available as alternatives to warfarin for stroke prevention in patients with atrial fibrillation. ${ }^{5}$ It should be emphasised that, unlike warfarin, the INR and aPTT are not very useful indicators of the anticoagulant effect of dabigatran, and pathology laboratories are currently establishing appropriate monitoring strategies for this new group of anticoagulants. ${ }^{6}$

Although perforation of a vessel is a rare but feared complication of mechanical thrombectomy, it is likely that this patient experienced an asymptomatic intimal tear with only transient extravasation in the context of repeated passes of the clot extraction device.

The Solitaire device used in our patient was originally designed for cerebral aneurysm bridging. However, a recent retrospective review of patients treated endovascularly with the Solitaire $\mathrm{AB}$ stent suggests that this procedure appears 
4 Non-contrast computed tomography scans after the endovascular procedure

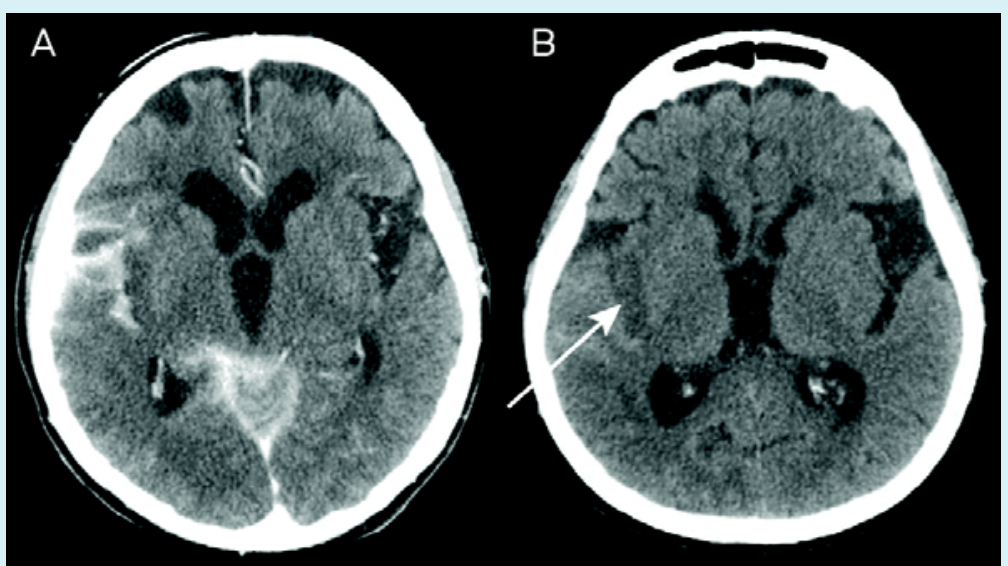

(A) Day 1 scan showing subarachnoid hyperdensity.

(B) Day 11 scan showing resolution of hyperdensity and residual infarction of the right posterior insular cortex (arrow).

to be safe and able to deliver favourable clinical outcomes in patients with proximal cerebral vessel occlusion, ${ }^{7}$ albeit without anticoagulation therapy. Currently, no guideline exists on the safety of treatments for acute stroke in patients taking dabigatran. Although there is anecdotal evidence of success with intravenous rtPA therapy in this setting, endovascular therapy holds promise because the risk of bleeding is theoretically lower. This is important because there is no practical immediate reversal of dabigatran currently available. There is preliminary evidence of the safety of endovascular therapy in acute stroke, ${ }^{7}$ however, data on its safety in patients taking anticoagulants are lacking. Such data would enable the development of a consensus guideline for this increasingly prevalent clinical problem.

Competing interests: No relevant disclosures.

Received 19 Dec 2011, accepted 15 Mar 2012.

1 Leyden JM, Chong WK, Kleinig T, et al. A population-based study of thrombolysis for acute stroke in South Australia. Med J Aust 2011; 194: 111-115.

2 De Smedt A, De Raedt S, Nieboer K, et al. Intravenous thrombolysis with recombinant tissue plasminogen activator in a stroke patient treated with dabigatran. Cerebrovasc Dis 2010; 30: 533-534.

3 Matute MC, Guillán M, García-Caldentey J, et al. Thrombolysis treatment for acute ischaemic stroke in a patient on treatment with dabigatran. Thromb Haemost 2011; 106: 178-179.

4 Lee L, Chryssidis S, Jannes J. Rescue therapy with local intra-arterial urokinase after poor clinical response with intravenous recombinant tissue plasminogen activator in acute ischaemic stroke. Intern Med J 2011; 41: 570-573.

5 Jaffer AK. Update in hospital medicine: studies likely to affect inpatient practice in 2011. Cleve Clin J Med 2011; 78: 430-434.

6 Favaloro EJ, Lippi G, Koutts J. Laboratory testing of anticoagulants: the present and the future. Pathology 2011; 43: 682-692.

7 Miteff F, Faulder KC, Goh AC, et al. Mechanical thrombectomy with a selfexpanding retrievable intracranial stent (Solitaire $A B$ ): experience in 26 patients with acute cerebral artery occlusion. Am J Neuroradiol 2011; 32: 1078-1081. 